\title{
Nuevos horizontes tras una Colaboración entre Artistas y Restauradores.
}

\section{New boundaries in Art after a collaboration between artists and restorers.}

TIPO DE TRABAJO:

Comunicación virtual.

PALABRAS CLAVE

conservación, artista contemporáneo, restaurador, colaboración, interdisciplinar

KEY WORDS

conservation, contemporary artist, art restorer, collaboration, interdisciplinary

\section{RESUMEN}

Las artistas Patricia Gómez y Mạ Jesús González son un colectivo artístico que mantiene una experimentación constante en su obra, no solo a nivel plástico, sino también a nivel conceptual. Como muchos otros artistas contemporáneos, han sucumbido a la experimentación de materiales y a ampliar los límites que la práctica artística tradicional establece, alcanzando con ello nuevos horizontes. La restauración ha sido el nuevo campo introducido en su obra. Desde una primera colaboración en 2014, han estado en constante contacto con especialistas en conservación y restauración con el objetivo de aumentar sus posibilidades técnicas y con ello, la imagen y el concepto final de su obra.

Este artículo expone los resultados y conclusiones obtenidos de las colaboraciones realizadas con las artistas entre 2014 y 2016 desde la perspectiva de las restauradoras implicadas, pero contando con la opinión de las artistas respecto a los cambios producidos en su concepción y obra como consecuencia de tal unión.

\section{ABSTRACT}

The artists Patricia Gomez and Maria Jesus Gonzalez are an artistic collective whose artwork is a mixture of experimentation techniques, both plastically and conceptually. As many contemporary artists, they have succumbed to the temptation of experimenting with new materials, exceeding the limits traditional art practice stablishes and so, reaching new boundaries. Restoration has been the new field introduced in their artwork. Since a first collaboration in 2014, Patricia and Maria Jesus have kept themselves in a constant contact with art conservation and restoration specialists. Their main purpose has been to increase their technical possibilities, in addition to the final appearance and concept of their artwork.

This paper will try to present the results and conclusions obtained from those collaborations made between artists and restorers from 2014 to 2016. All the information will be presented from the restorers' perspective, taking account of the opinions of the artists, in order to determinate the changes in their artwork conception as consequence of the collaboration. 


\section{CONTENIDO.}

\section{INTRODUCCIÓN.}

Podría decirse que la interdisciplinariedad en la producción artística contemporánea es un fenómeno cotidiano. Los artistas, en su continua búsqueda de nuevas formas de expresión, indagan más allá de los límites que se les presentan, aventurándose en territorios desconocidos para el Arte. La Restauración aparece en el horizonte como uno de estos destinos que empiezan a ser explorados, como demuestra la obra del artista -también arquitecto y restaurador- Jorge Otero Pailos, modelo en sí mismo de la fusión de disciplinas en el mundo del Arte con su serie The Ethics of Dust (Zyman, 2010; Otero-Pailos, 2017).

Otro ejemplo de esa interdisciplinariedad es el colectivo del que se ocupa este artículo, el formado por las artistas valencianas Patricia Gómez y Ma Jesús González. Estas artistas trabajan desde 2002 el concepto de la memoria abandonada que reside en la piel de ciertos edificios. Con la intención de preservar, extraen las vivencias que sus paredes transmiten y que, de otro modo, caerían en el olvido.

La técnica artística que utilizan se acerca mucho a la concepción de los arranques murales empleados en restauración, aunque difiere en los materiales, ya que su objetivo prioritario no es la conservación fiel de los estratos arrancados sino la permanencia conceptual del recuerdo de los individuos que habitaron esos lugares. A esa técnica tan propia, las artistas la denominan "estampación por arranque".

Debido a su método de trabajo y a su apertura de miras hacia nuevas soluciones técnicas, frecuentemente han consultado a especialistas en el uso de materiales específicos o han experimentado procesos con ellos. De esta manera, en 2014, para el proyecto artístico sobre las rutas migratorias que estaban iniciando en ese momento en el centro de internamiento de extranjeros de Fuerteventura, À tous les Clandestins ${ }^{1}$, decidieron contar con la ayuda de una restauradora dentro de su equipo de trabajo. Aunque, inicialmente, la presencia de la especialista se planteó solo como apoyo, los problemas surgidos durante el proceso creativo dieron lugar a una colaboración más amplia. En esta, se propuso la sustitución de su técnica habitual por la propia del campo de la restauración denominada strappo, como solución para las zonas que presentaban problemas (Fuentes Durán, 2016:47-49).

Las artistas, de la mano del equipo de especialistas en restauración, aprendieron la técnica del arranque a strappo desde un planteamiento práctico, pudiendo experimentar en conjunto con todos los procedimientos de preparación, aplicación y recuperación de los arranques, tanto en Fuerteventura, como en los procedimientos posteriores, ejecutadas en su taller en Valencia.
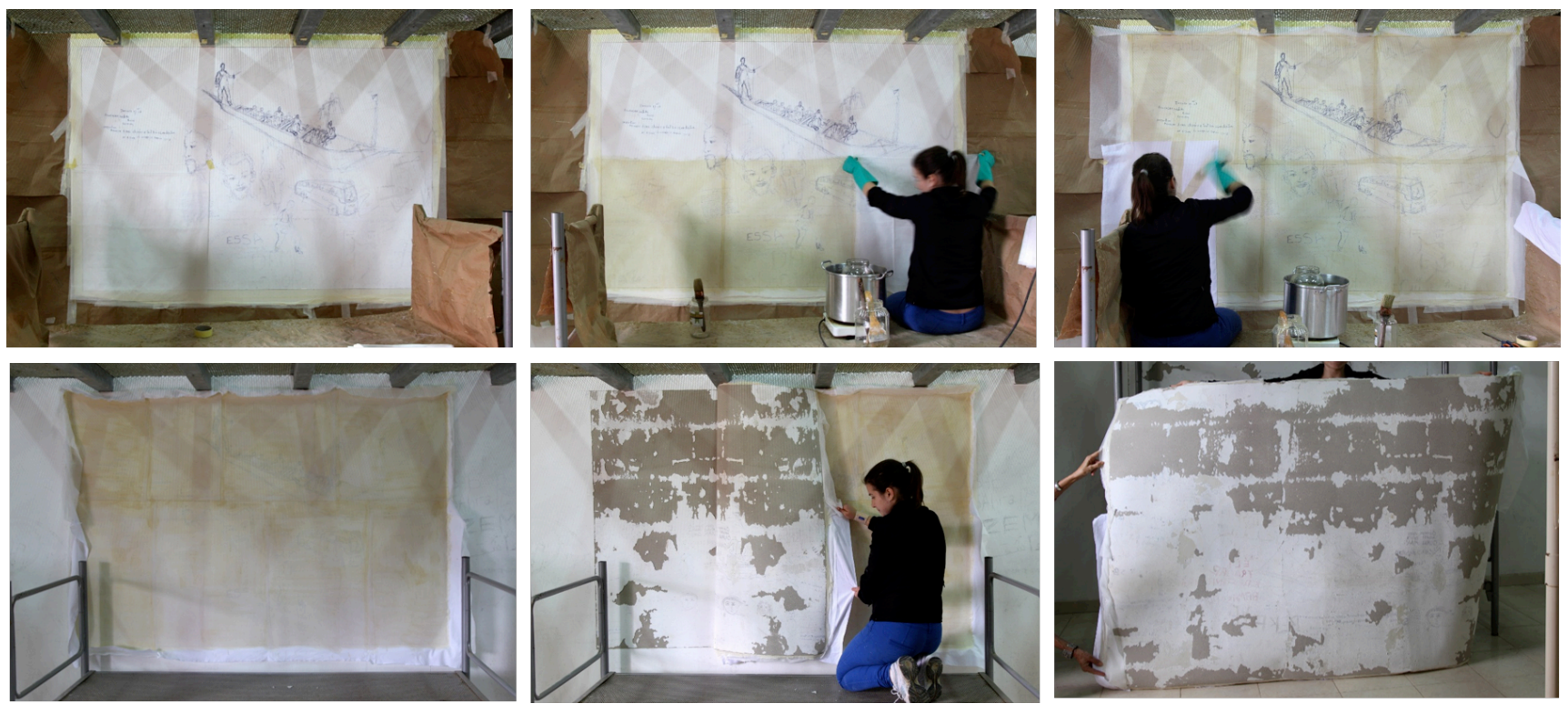

Ilustración 1. Proceso de arranque a strappo en CIE Fuerteventura. R.L. Amor ${ }^{\odot}$

\footnotetext{
${ }^{1}$ À tous les Clandestins es un proyecto premiado con la Beca Endesa para Artes Plásticas, centrado en la recuperación de la memoria de los inmigrantes que estuvieron retenidos en dos centros de internamiento de extranjeros (CIEs de Fuerteventura y Nuadibú) a través del rescate de los escritos y dibujos que quedaron en sus paredes (Gómez, 2017).
} 
Amor García, Rita Lucía; Fuentes Durán, Eva Mariana; Soriano Sancho, Mạ Pilar

Nuevos Horizontes tras una Colaboración entre Artistas y Restauradores

III CONGRESO INTERNACIONAL DE INVESTIGACIÓN EN ARTES VISUALES :: ANIAV 2017 :: GLOCAL [codificar, mediar, transformar, vivir] http://dx.doi.org/10.4995/ANIAV.2017.4883

La utilización del arranque a strappo permitió resolver los problemas puntuales que obstaculizaban el progreso de su trabajo, pero, además, las características de las piezas resultantes han abierto una nueva vía en la experimentación de estas creadoras, que han continuado utilizando la técnica como recurso artístico.

\section{DESARROLLO Y AMPLIACIÓN DE LA COLABORACIÓN.}

Esa primera colaboración supuso un cambio en sus planteamientos artísticos siguientes, un enriquecimiento para su obra que les abría camino hacia nuevas soluciones en su característica forma de trasladar mensajes desde lugares remotos o en vías de extinción, al público. Su particular "estampación por arranque" se alternaba con técnicas de arranque de restauración y, consecuentemente, aportaba nuevas ideas y formas para la obra final.

Así fue como, justo después de completar su estancia en Fuerteventura, Patricia y Ma Jesús continuaron su proyecto sobre la inmigración en un segundo emplazamiento. Como complemento al trabajo realizado en el centro de internamiento de extranjeros de la isla, se trasladaron a Nuadibú, Mauritania, fijando su base de investigación en el centro de internamiento de la ciudad. Este fue, para muchas personas, el lugar donde su sueño de encontrar una nueva vida en Europa quedaba truncado, antes siquiera de atravesar los lindes dentro de África.

Al tratarse del mismo proyecto, las artistas mantuvieron un nexo de unión entre lo realizado en Fuerteventura y el trabajo en Nuadibú. En cambio, el planteamiento de creación en esta segunda ubicación no contó con un restaurador in situ. Las artistas se sentían seguras de poder aplicar por sí solas el sistema aprendido durante su colaboración en Fuerteventura, habiendo entendido el consecuente trabajo que suponía el arranque a strappo, en comparación con el uso de su técnica. Además de emplear el procedimiento de restauración para la obtención de los fragmentos murales en esta fase del proceso creativo, las artistas plantearon y obtuvieron piezas de mayores dimensiones a aquellas recogidas en Fuerteventura mediante el mismo sistema.

Este proyecto resultaba interesante desde diferentes perspectivas: a nivel artístico, por la continuidad conceptual y material en su trabajo con la nueva técnica; y a nivel de restauración, por la inclusión de una técnica conservativa dentro del repertorio de posibilidades artísticas del colectivo.

Por otro lado, la dificultad que entrañaba el proceso de arranque, y, sobre todo, los trabajos posteriores en el taller, incrementaba el periodo de colaboración entre artistas y restauradores, lo cual se presentaba beneficioso para todos los intervinientes. Para las artistas, que la colaboración continuara resultaba enriquecedor según las nuevas posibilidades que se les ofrecía, y mayor seguridad de cara a la conservación de sus obras. Para los restauradores era una manera de seguir observando y recogiendo datos sobre los procedimientos y aspectos creativos de la producción plástica de Patricia y María Jesús.

En el último proyecto en el que han participado, La casa Ena ${ }^{2}$, en Huesca, trabajaron en el edificio donde el artista y pedagogo anarcosindicalista Ramón Acín Aquilué vivió, junto a su familia, hasta su fusilamiento el 6 de agosto de 1936 por los militares sublevados a la República. La casa pasó después por otras vicisitudes, siendo incluso, durante un tiempo, almacén de juguetes.

En este proyecto, el uso del arranque a strappo no se debió a una necesidad formal o de continuidad, sino que las artistas decidieron combinar su técnica y la de restauración con una intención únicamente artística y conceptual. En concreto, utilizaron una combinación de ambas técnicas en las diferentes superficies de la casa como medio de obtención de una estratigrafía de la pared en varios momentos de la vida del edificio, como alusión a las diferentes etapas de la nutrida historia de ese lugar.

La técnica de las artistas, la estampación por arranque, se aplicaba a los estratos más superficiales de las paredes, la cual recogía los recuerdos más inmediatos de la casa. Al mismo tiempo, en espacios próximos, las artistas aplicaban el arranque a strappo, extrayendo fragmentos más profundos de los soportes, y con ello, de la historia de la casa y sus habitantes.

\footnotetext{
${ }^{2}$ La Casa Ena es un proyecto colectivo bajo la propuesta de Sara Álvarez Sarrat, premiado con la Beca Ramón Acín 2016 desarrollado entorno a la casa y vida de Ramón Acín Aquilué (DP Huesca, 2016).
} 

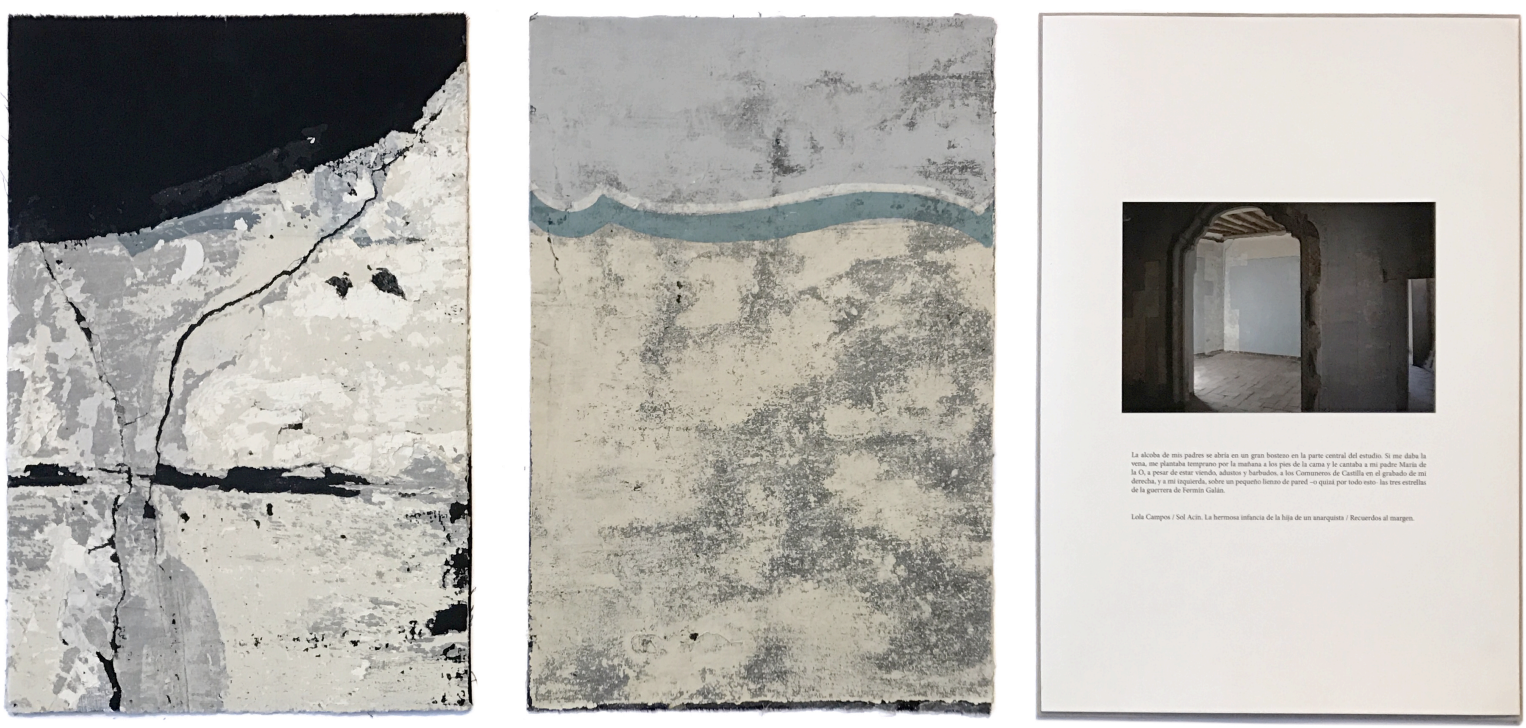

llustración 2. Resultado de las técnicas utilizadas en La Casa Ena. M.J. González/P. Gómez ${ }^{\circledR}$

En Huesca, al igual que en Nuadibú, las artistas, experimentadas ya en la ejecución y dificultades que el arranque a strappo entraña, realizaron los arranques dirigidas por sí mismas, prescindiendo nuevamente de un restaurador in situ. A pesar de ello, el apoyo presentado por los especialistas y su implicación durante las diferentes etapas del proceso creativo es una de las cuestiones que más gustan a las artistas, por lo que este proyecto se planteó como una colaboración puntual entre las artistas y estudiantes de la Escuela Superior de Conservación y Restauración de Bienes Culturales de Huesca. En este caso, los estudiantes realizaron tareas de asistencia más focalizadas hacia soluciones que las artistas ya conocían y esperaban de antemano, como una performance colaborativa entre dos disciplinas diferenciadas.

Por tanto, como hemos visto, tanto en Nuadibú como en Huesca, las artistas han utilizado el strappo sin ayuda de un restaurador in situ, siguiendo las recomendaciones formales y técnicas aprendidas en el primer proyecto de colaboración. A pesar de que hayan asimilado la técnica, la colaboración no ha cesado, ya que los procesos posteriores al arranque -como la eliminación del cemento del reverso, el refuerzo del estrato y la retirada de los materiales del encolado sobre el anverso- se han llevado a cabo junto a las restauradoras. Estas últimas también han intervenido aplicando medidas de emergencia ante problemas presentados en algunas obras una vez arrancadas -como descohesión puntual o presencia de microorganismos por cambio de temperatura y humedaddifíciles de solucionar por las propias artistas.

\section{COMPLEMENTO A LA COLABORACIÓN: ENTREVISTAS.}

Además de toda la información y datos relevantes (acerca de materiales, procesos, tiempos, criterios, etc.) que aporta un contacto tan directo y constante con las artistas durante la creación de su obra, hemos querido dejar testimonio de cómo han percibido ellas esta experiencia a través de varias entrevistas a lo largo y al final de la colaboración llevada a cabo entre 2014 y 2016.

Pese a que la mayoría de cuestiones tratadas en las entrevistas eran ya conocidas por los restauradores gracias a ese contacto con las artistas, siguiendo lo propuesto por autores especializados en este campo de investigación como Lydia Berkens (2012), no se quiso obviar la realización de estas, por su importancia de cara a organizar y reforzar algunas ideas y como testimonio documental de las ideas de las artistas. 


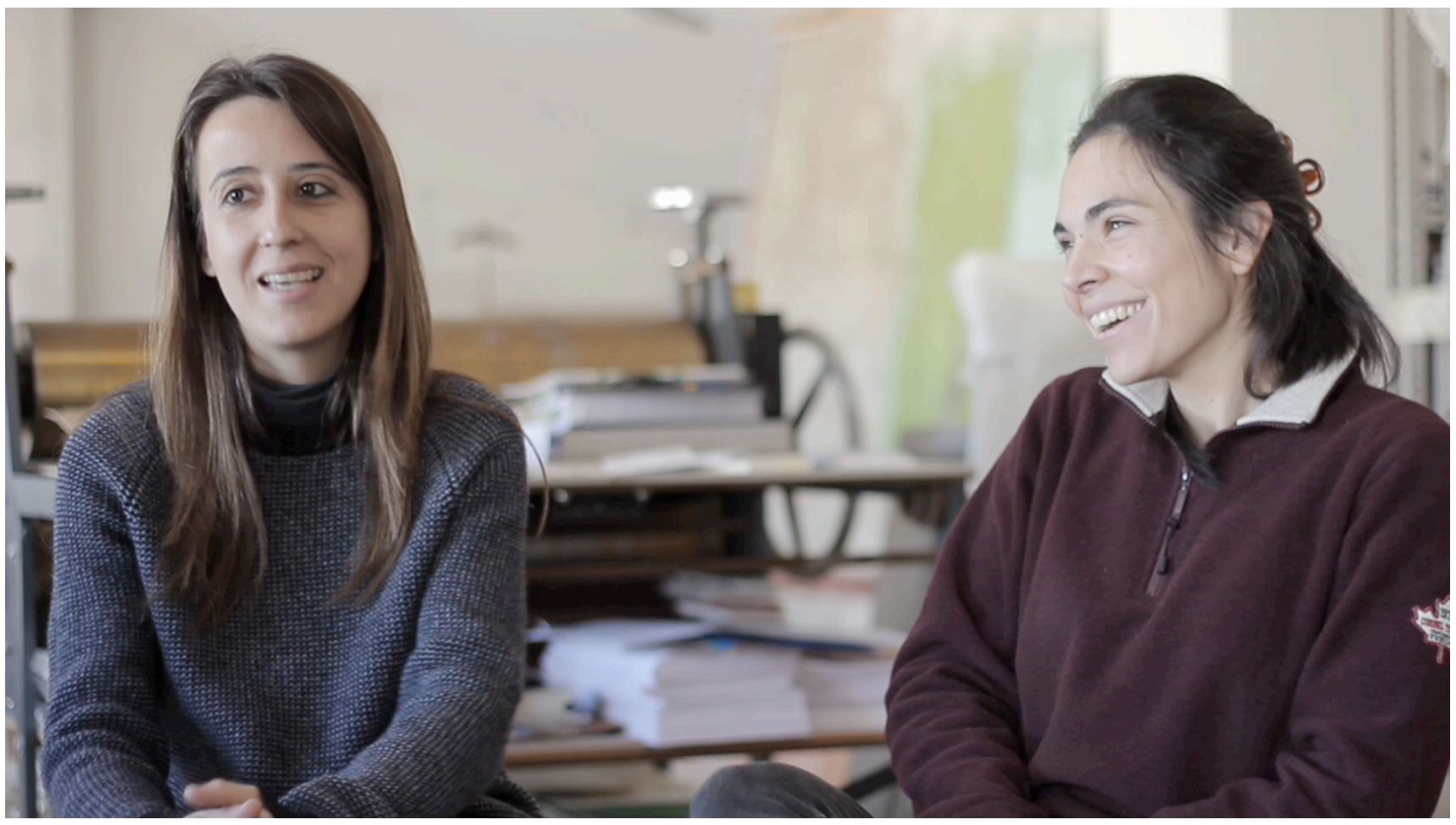

llustración 3. Entrevista con las artistas en su estudio. R.Fuentes ${ }^{\circledR}$

De forma general las entrevistas tenían como objetivo conocer dos áreas diferentes: las ideas de Patricia y Mạ Jesús sobre su arte en general, así como las relativas a los aspectos específicos de cada proyecto.

Dentro del primer objetivo, resultaba básico preguntarles acerca de los materiales que usan frecuentemente para identificarlos y saber qué características valoran en ellos; o, lo que es lo mismo, por qué los usan. La utilidad de esta información reside en conocer la importancia y prioridad de cada elemento en su obra. En las colaboraciones, en concreto, nos ha servido para proponer el empleo de materiales alternativos que, aportando unas mejores características de cara a la conservación y el envejecimiento, ofrecieran las cualidades estéticas y plásticas semejantes a las de los materiales empleados habitualmente por las artistas. Documentar estos aspectos también resulta útil a la hora de plantear la conservación preventiva o una posible restauración de su obra si no se pudiera contar con la opinión de las artistas.

En relación con la conservación, también se han querido conocer sus ideas respecto al envejecimiento y deterioro o degradación de sus piezas artísticas. Si bien las artistas se mueven en un campo conceptual asociado a la memoria y, por tanto, el paso del tiempo es algo muy ligado a su obra, no buscan el deterioro, sino poder con ellas recuperar los testimonios parietales que van a desaparecer. Sus opiniones respecto a la obra que ellas crean fluctúan entre la aceptación del envejecimiento y la voluntad de pervivencia. Estas vacilaciones se deben a que, según sus propias palabras, desconocen "cómo podría ser la degradación de su obra" aunque sí tienen claro su rechazo a "la reconstrucción completa y artificial" de una pieza en caso de un gran deterioro.

Ligado a esto y como se habrá podido comprender con el apartado anterior, su posición respecto a la restauración y los restauradores es de máximo respeto además de una completa apertura tanto a compartir sus técnicas como a aprender de este campo.

El último aspecto que queremos destacar respecto a lo obtenido con las entrevistas hace referencia a sus consideraciones acerca del empleo del arranque a strappo. Durante el primer proyecto consideran que es algo puntual marcado por la necesidad (dificultad para realizar los arranques con su propia técnica); en cambio, en su siguiente proyecto La casa Ena lo vuelven a utilizar ya de forma voluntaria, no llevadas por la necesidad, combinándolo con su propia técnica de "estampación por arranque" como una forma de seguir avanzando en su camino creativo.

En la actualidad se encuentran en el proceso de estudio teórico de sus próximos proyectos, para el que ya han manifestado su voluntad de contar, de nuevo, con la colaboración de las restauradoras. En estos, Patricia y $\mathrm{M}^{a}$ Jesús plantean nuevas metas que alcanzar y nuevos aspectos que pulir en la colaboración con especialistas en restauración. 


\section{RESULTADOS.}

La utilización del arranque a strappo permitió resolver los problemas puntuales que obstaculizaban el progreso de su trabajo, pero, además, las características de las piezas resultantes han abierto una nueva vía en la experimentación de estas artistas, y consecuentemente, en las obras expuestas a posteriori.

Hasta el momento, los resultados de esta colaboración continua y activa se han visto representados y accesibles al público por primera vez, en tres exposiciones organizadas entre 2016 y 2017, y también, en la feria de arte contemporáneo ARCO 2017.

La primera en celebrarse fue La casa Ena, exposición colectiva en la Sala de Exposiciones de la Diputación de Huesca (17 de septiembre al 23 de octubre de 2016) que aunaba proyectos relacionados de diferentes artistas, los cuales exploraban diversos aspectos del último periodo de vida de Ramón Acín Aquilué. La obra presentada por Patricia y Mã Jesús, se centraba en la memoria retenida en la casa de Ramón Acín y su familia.

La segunda exposición, titulada À tous les clandestins, estaba vinculada directamente al proyecto con el mismo nombre. Esta exposición fue inaugurada en el Museo de Teruel el 17 de noviembre de 2016 hasta el 19 de febrero del 2017, como parte final de la Beca Endesa para Artes Plásticas que habían recibido las artistas para subvencionar el proyecto.

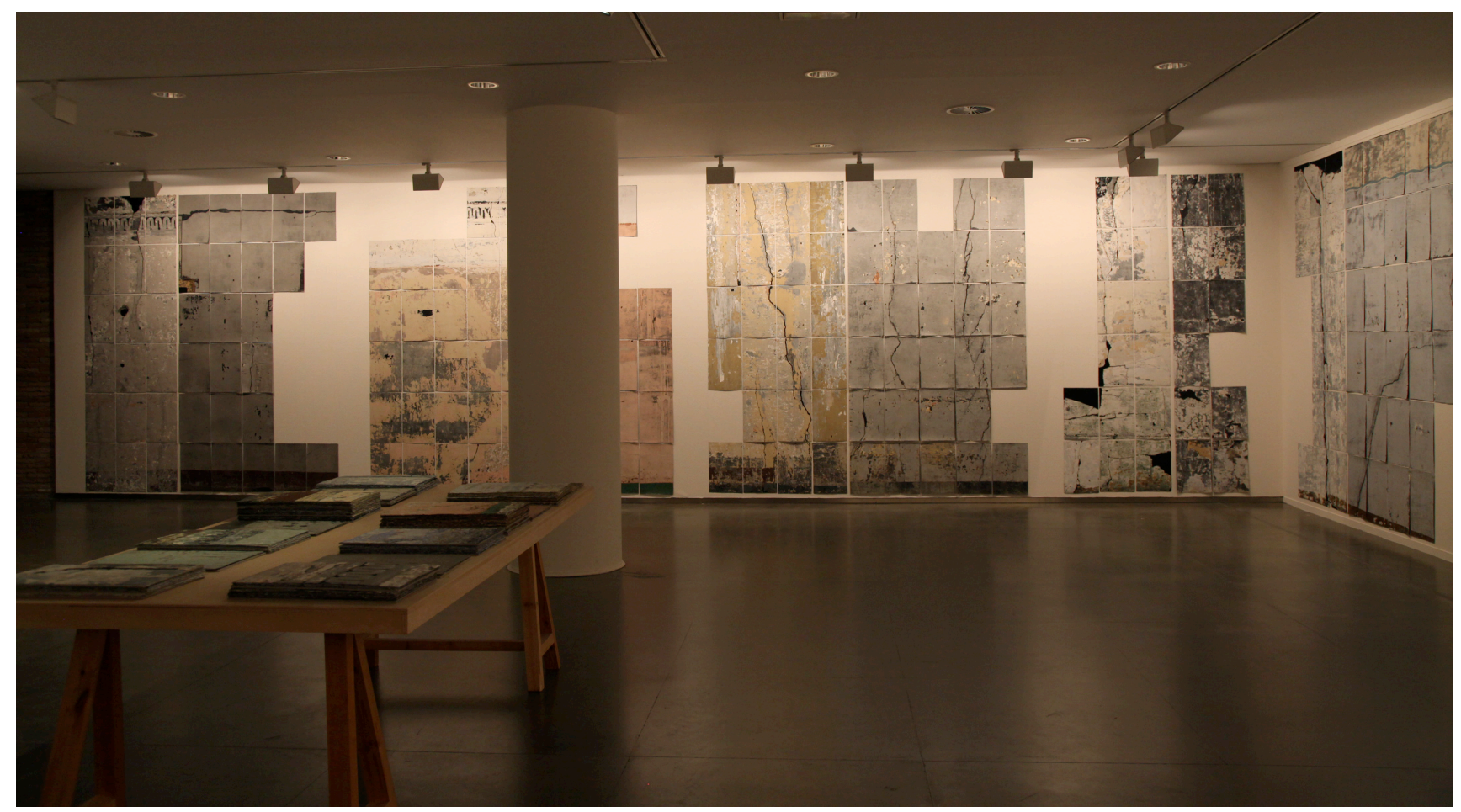

Ilustración 4. Exposición en la Diputación Provincial de Huesca. M.J. González/P. Gómez ${ }^{\circledR}$ 


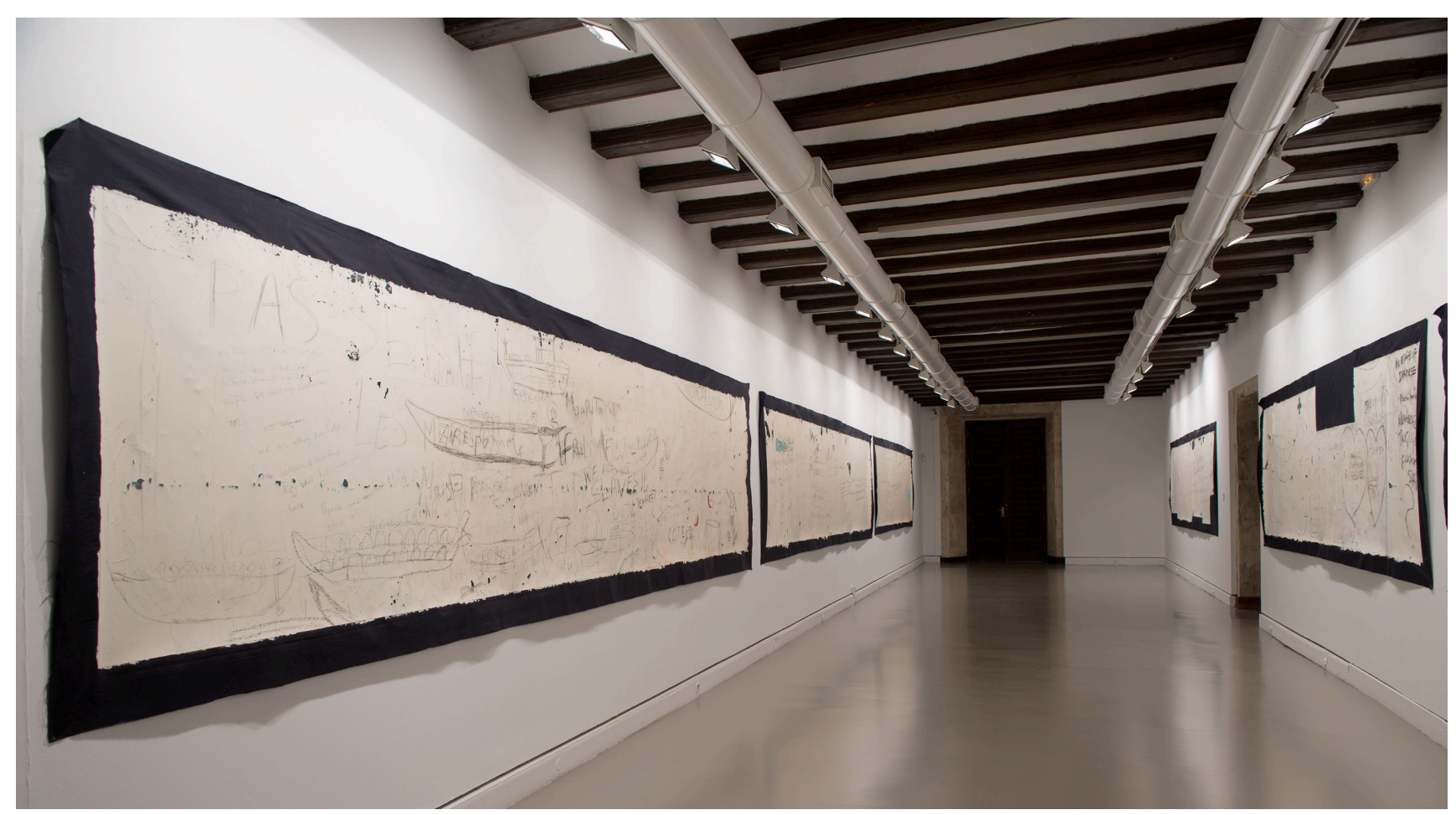

Ilustración 5. Exposición en el Museo de Teruel. M.J. González/P. Gómez ${ }^{\odot}$

En estas dos exposiciones, las artistas han creado montajes en los que el contraste entre las dos técnicas (la originalmente suya y la aprendida) son vehículos discretos y efectivos para transmitir la memoria de las personas que habitaron esos espacios, tan diferentes entre sí, pero, en cualquier caso, intensamente vividos.

Por último, la tercera exposición realizada contaba con la exhibición de una obra en particular extraída del proyecto À tous les Clandestins realizada a partir de esa colaboración. En esta ocasión, se trató de una exposición conjunta titulada El borde de una Herida. Migración, exilio y colonialidad en el estrecho, en el centro cultural CentroCentro Cibeles de Cultura y Ciudadanía de Madrid, expuesta del 17 de febrero al 4 de junio de 2017 (CentroCentro, 2017).

Al mismo tiempo, su objetivo es poner de manifiesto la importancia de la obra artística y el proceso de su obtención, por lo que, ahora, como obras de arte no solo se presentan las piezas arrancadas, también las filmaciones del proceso artístico. Así se le da importancia al procedimiento del arranque a strappo como la manera de desnudar esas paredes donde quedaba esa memoria. La idea de conservación del concepto y de la materia es el centro de atención en estas grabaciones, que, como arranques de restauración, siguen un procedimiento correcto con: la obra, en el mantenimiento de la misma y el aumento de vida; el autor, en la difusión de su memoria; y, el público, el cual estaba limitado en la apreciación de esas formas.

\section{CONCLUSIONES.}

Las colaboraciones entre artistas y especialistas de otras disciplinas no son un hecho aislado en el arte, pero todavía se requiere un aumento en su exploración. Acercamientos como este suponen un nuevo cambio de entendimiento de los papeles de conservadores y artistas en el arte contemporáneo, donde los límites tradicionales se transforman y se abre paso a nuevos horizontes. Además, estas colaboraciones son, para los artistas, una apertura de posibilidades en la producción artística; y para los restauradores, un mecanismo de obtención de información, que, de otro modo, sería inalcanzable.

En este punto, el proceso creativo y la colaboración ligada a él han terminado: los proyectos están expuestos, de modo que las obras ya han comenzado su andadura. $Y$, con esta andadura, comienza otro periodo de trabajo conjunto en el que artistas y conservadoresrestauradores seguirán colaborando. 
Amor García, Rita Lucía; Fuentes Durán, Eva Mariana; Soriano Sancho, Mạ Pilar

Nuevos Horizontes tras una Colaboración entre Artistas y Restauradores

III CONGRESO INTERNACIONAL DE INVESTIGACIÓN EN ARTES VISUALES :: ANIAV 2017 :: GLOCAL [codificar, mediar, transformar, vivir] http://dx.doi.org/10.4995/ANIAV.2017.4883

\section{FUENTES REFERENCIALES.}

BERKENS, L. et al. (2012). The Artist Interview for Conservation and Presentation of Contemporary Art. Guidelines and Practice. Amsterdam: Jap Sam Books.

FUENTES DURÁN, E; AMOR GARCÍA, R.L. y SORIANO SANCHO, M.P. (2016). "La colaboración artista-restaurador durante el proceso creativo:À tous les Clandestins de Patricia Gómez y María Jesús González". En Conservación de Arte Contemporáneo, $17 \underline{a}$ Jornada. Madrid: Museo Nacional Centro de Arte Reina Sofía.

ZYMAN, D.; BIRNBAUM, D.; VON HABSBURG, F. (2010). Jorge Otero-Pailos: The Ethics of Dust. Colonia: Thyssen Bornemisza Art Contemporary.

CENTROCENTRO. (2017). “EL BORDE DE UNA HERIDA. Migración, exilio y colonialidad en el Estrecho”. En CentroCentro Cibeles de Cultura y Ciudadanía. <http://www.centrocentro.org/centro/exposicion_ficha/222> [Consulta: 5 de marzo de 2017]

GÓMEZ, P; GONZÁLEZ, M.J. (2017). “À tous les Clandestins”. En Patricia Gómez y María Jesús González. <http://www.patriciagomezmariajesusgonzalez.com/A-tous-les-clandestins> [Consulta: 5 de marzo de 2017]

DIPUTACIÓN PROVINCIAL DE HUESCA. (2016). "Sara Álvarez Sarrat. La Casa Ena. Beca Ramón Acín 2016". En Diputación de Huesca, Sala de Exposiciones. <http://www.dphuesca.es/oferta-cultural/exposicion-sala-dph/-/publicador/exposicion-sara-alvarez-becaramon-acin-2016/WI2Bry594pBV?p_p_auth=f3eEgpLb\&p_p_state=maximized> [Consulta: 5 de marzo de 2017]

STUDIO OTERO-PAILOS. (2017). "The Ethics of Dust Series". En JORGE OTERO-PAILOS Artist. Architect. Preservationist. <http://www.oteropailos.com/the-ethics-of-dust-series/> [Consulta: 5 de marzo de 2017] 